\title{
CARATHÉODORY CONVEX SELECTIONS OF SET-VALUED FUNCTIONS IN BANACH LATTICES
}

\author{
JERZY MOTYL
}

\begin{abstract}
Let $T$ be a measurable space, $X$ a Banach space while $Y$ a Banach lattice. We consider the class of "upper separated" set-valued functions $F: T \times X \rightarrow 2^{Y}$ and investigate the problem of the existence of Carathéodory type selection, that is, measurable in the first variable and order-convex in the second variable.
\end{abstract}

\section{Introduction}

In general, investigating deterministic or stochastic inclusions an appropriate kind of regularity of their multivalued structure is required. Regular selections have attracted considerable interest as a useful tool for proving the existence of solutions of such inclusions. The investigation of Carathéodory selections of multifunctions (i.e. selections which are measurable in the first variable and continuous in second one) was initiated by Castaing [3], Cellina [4] and Fryszkowski [8], and continued among others by Ioffe [11], Ricceri [18], Rybiński [19], Kucia [13], Fierro, Martinez and Morales [7]. The existence of classical Carathéodory selections, so valuable in the theory of differential inclusions is not appriopriate enough in the investigation of stochastic inclusions. Such problems require the existence of more regular selections. Hermes [9], Belov and Chistyakov [2] and Chistyakov [5] treated problems of the existence of selections of multifunctions

2010 Mathematics Subject Classification. 22B15, 28B20, 54C65.

Key words and phrases. Banach lattice, upper separated multifunction, Carathéodory selection, convex selection. 
of one real variable which are of bounded variation, Lipschitz or absolutely continuous. Using these results Nowak and Chistyakov in [6] discussed the existence of Carathéodory type selections which are measurable in the first variable and preserve the regularity mentioned above with respect to their second variable.

Motyl and Michta in [15] introduced the new class of multifunctions taking their values in Banach lattices and called "upper separated". They proved that such multifunctions admit order-convex and locally Lipschitz selections.

In the paper we study the problem of the existence of the Carathéodory type selections being measurable in the first variable and order-convex and locally Lipschitz in the second variable.

\section{Notation and preliminary facts}

We begin our considerations with auxiliary definitions and facts needed in the sequel.

Let $X$ be a Banach space and let $Y$ be a Banach lattice. Let $K^{+}$denote a cone of positive elements in $Y$. We will use the notation $x \preceq y$ if $y-x \in K^{+}$. $(Y, \preceq)$ is an order-complete if every nonempty and majorized subset of $Y$ has a supremum in $Y$. A set $A$ in a Banach lattice is called order-bounded if it is contained in some order interval $[a, b]=\{y \in Y: a \preceq y \preceq b\}$. A set $A$ in a Banach lattice is called order-convex (or full) if for each $x, y \in A$ the order interval $[x, y]$ is contained in $A$.

We adjoin to $Y$ the greatest element $+\infty$ together with the lowest element $-\infty$ and extend the vector space operations in a natural way. Let $\bar{Y}=Y \cup\{ \pm \infty\}$.

Let us consider an extended function $f: X \rightarrow \bar{Y}$. Let $\operatorname{Dom} f=\{x \in X$ : $f(x) \neq \pm \infty\}$ and define the epigraph of $f$ by the formula

$$
\operatorname{Epi}(f)=\{(x, a) \in X \times Y: f(x) \preceq a\} .
$$

Definition 2.1. A function $f: X \rightarrow \bar{Y}$ is order-convex if for every $x, y$ in $X$ and $\lambda \in[0,1]$

$$
f(\lambda x+(1-\lambda) y) \preceq \lambda f(x)+(1-\lambda) f(y) .
$$

A function $f: X \rightarrow \bar{Y}$ is locally order-Lipschitz if and only if for every $x_{0} \in X$ there exist an open neighbourhood $U_{x_{0}}$ and $y \in K^{+}$such that

$$
|f(x)-f(z)| \preceq y\|x-z\| \quad \text { for every } x, z \in U_{x_{0}} .
$$

A set-valued function $F: X \rightarrow 2^{Y}$ is majorized in a neighbourhood of $x_{0}$ if there exist an open neighbourhood $U_{x_{0}}$ and $y \in Y$ such that for each $x \in U_{x_{0}}$ and every $a \in F(x)$ the inequality $a \preceq y$ holds.

Let $\mathrm{Cl} Y$ (resp. Comp $Y$, Conv $Y$ ) denote the family of all closed, (resp. compact, convex) and nonempty subsets of $Y$.

$$
\text { Comp Conv } Y:=\operatorname{Comp} Y \cap \operatorname{Conv} Y, \quad \operatorname{Cl} \operatorname{Conv} Y:=\operatorname{Cl} Y \cap \operatorname{Conv} Y \text {. }
$$


By $\mathcal{L}(X, Y)$ we denote the space of all linear and continuous operators from $X$ to $Y$.

A set-valued function $F$ from the measurable space $(T, \mathcal{M})$ to $\mathrm{Cl} Y$ is said to be measurable if $F^{-1}(U)=\{t \in T: F(t) \cap U \neq \emptyset\} \in \mathcal{M}$ for every open subset $U$ of $Y$.

A set-valued function $F: X \rightarrow \mathrm{Cl} Y$ is called lower semicontinuous (lsc) if $F^{-1}(U)$ is open for every open subset $U$ of $Y$. If $F^{-1}(V)$ is closed for every closed set $V \in Y$, then $F$ is called upper semicontinuous (usc). $F$ is continuous if it is usc and lsc.

A function $f: X \rightarrow Y$ is called a selection of $F$ if $f(x) \in F(x)$ for every $x \in X$.

REMARK 2.2. Since the Banach lattice $Y$ admits two structures, order and norm, we use in the paper the following convention: all properties like closedness, convexity, compactness, continuity etc. mean properties with respect to the norm, while properties with respect to the order or order-topology are denoted by the symbol "order-property".

Let $V, W: X \rightarrow \bar{Y}$ be defined by formulas $V(x)=\sup \{a: a \in F(x)\}$ and $W(x)=\inf \{b: b \in F(x)\}$.

Let $\Pi_{F(x)}(a)$ denote the metric projection of a point $a \in Y$ onto the set $F(x)$. We define

$$
\begin{gathered}
\bar{V}(x):= \begin{cases}\Pi_{F(x)}(V(x)) & \text { for } x \in \operatorname{Dom} V, \\
+\infty & \text { for } x \notin \operatorname{Dom} V,\end{cases} \\
\bar{W}(x):= \begin{cases}\Pi_{F(x)}(W(x)) & \text { for } x \in \operatorname{Dom} W, \\
-\infty & \text { for } x \notin \operatorname{Dom} W .\end{cases}
\end{gathered}
$$

Definition 2.3. A set-valued function $F: X \rightarrow \mathrm{ClConv} Y$ is upper separated if each point $(x, \bar{W}(x)-\varepsilon)$ can be separated from the set $\operatorname{Epi}(\bar{V})$ in the following sense: for every $x \in X$ and each $\varepsilon \in K^{+} \backslash\{0\}$ there exist $A \in \mathcal{L}(X, Y), a \in \mathbb{R}^{1}$ and $\delta \in K^{+} \backslash\{0\}$ such that for every $y \in \operatorname{Dom} \bar{V}$ and each $b \in K^{+}$the condition

$$
A(x)-A(y)+a(\bar{W}(x)-\bar{V}(y)-\varepsilon-b)-\delta \in K^{+}
$$

holds.

EXAmPLe 2.4. Let $X=C\left([a, b], \mathbb{R}^{1}\right)$ and let $Y$ be an arbitrary order complete Banach lattice with a positive cone $K^{+}$. $\operatorname{Var}(x)$ denotes a total Jordan variation of the function $x$ on the interval $[a, b]$. Let $z \in K^{+} \backslash\{0\}$ be arbitrary fixed. We define a set-valued function $F: C[a, b] \rightarrow 2^{Y}$ by the formula:

$$
F(x)= \begin{cases}{[0, z]} & \text { for } x \text { such that } \operatorname{Var}(x)<\infty, \\ {[-z, 0]} & \text { for } x \text { such that } \operatorname{Var}(x)=\infty .\end{cases}
$$


Observe that $V(y)$ is equal to $z$ for $\operatorname{Var}(y)<\infty$ and takes on the value 0 otherwise. Similarly $W(x)$ takes on 0 or $-z$ as its values. Therefore, taking $A \equiv 0, a=-1, \delta=\varepsilon$ in Definition 2.3, and noting that $\bar{W}(x)=W(x)$, $\bar{V}(y)=V(y)$ we obtain the inequality

$$
\forall x, y \in C\left([a, b], \mathbb{R}^{1}\right) \forall b \in K^{+} \quad V(y)+b \succeq W(x) .
$$

which is clearly fulfiled because of

$$
V(y)+b \succeq \min \{z, 0\} \succeq \max \{0,-z\} \succeq W(x) .
$$

This means that $F$ is upper separated. Since finite variation functions and infinite variation functions form dense subsets of $C\left([a, b], \mathbb{R}^{1}\right)$ the above defined $F$ is neither upper nor lower semicontinuous in any point $x \in C\left([a, b], \mathbb{R}^{1}\right)$.

Let $Z$ be a locally compact metrizable and separable topological space. By $C(Z, Y)$ we denote the space of all continuous functions from $Z$ to $Y$ endowed with the topology of of the uniform convergence on compact subsets of $Z$. Then $C(Z, Y)$ is a Polish space. Let $\beta(C(Z, Y))$ denote the $\sigma$-field of Borel measurable subsets of $C(Z, Y), \beta(Z)$ and $\beta(Y)$ denote the Borel $\sigma$-fields of subsets of $Z$ and $Y$, respectively.

Definition 2.5. Let $\mathcal{N}^{*}$ and $\mathcal{N}$, respectively, denote the sets of all finite and infinite sequences of positive integers. A family $\mathcal{M}$ of sets satisfies Suslin property if for each function $f: \mathcal{N}^{*} \rightarrow \mathcal{M}$ the set $\bigcup_{\sigma \in \mathcal{N}} \bigcap_{n=1}^{\infty} f\left(\sigma_{1}, \ldots, \sigma_{n}\right)$ belongs to $\mathcal{M}$.

RemarK 2.6. Let $(T, \mathcal{M})$ be a measurable space. If $\mathcal{M}$ satisfies Suslin property then for each Polish space $Z$ and each $A \in \mathcal{M} \otimes \beta(Z)$ its projection $\Pi_{T}(A)$ belongs to $\mathcal{M}$. Moreover, let us note that every complete (with respect to a $\sigma$ finite measure) $\sigma$-field $\mathcal{M}$ satisfies Suslin property.

Definition 2.7. A set-valued function $F: T \times Z \rightarrow \mathrm{Cl} Y$ is Carathéodory if $F(\cdot, z)$ is $\mathcal{M}$-measurable for all $z \in Z$ and $F(t, \cdot)$ is continuous for every $t \in T$.

We will need the following results in the sequel:

Theorem 2.8 ([15]). Let $F: X \rightarrow \mathrm{ClConv} Y$. If $F$ is upper separated, then there exists an order-convex function $f: X \rightarrow \bar{Y}$ such that $f(x) \in F(x)$ in each point $x$ for which the set $F(x)$ is order-bounded and order-convex.

TheOREm 2.9 ([14]). Let $(T, \mathcal{M})$ be a measurable space satisfying Suslin property, let $Z$ be a Polish space and $F: T \rightarrow 2^{Z}$ a set-valued function. If Gr $F$ is $\mathcal{M} \otimes \beta(Z)$ measurable, then $F$ has a measurable selector. 
Theorem $2.10([13])$. Let $(T, \mathcal{M})$ be a measurable space, let $Z$ be a locally compact metrizable and separable topological space and $Y$ a separable Banach space. If $\phi$ from $T \times Z$ into closed and convex subsets of $Y$ is Carathéodory, then $\phi$ is $\mathcal{M} \otimes \beta(Z)$-measurable.

Lemma $2.11([8])$. Let $(T, \mathcal{M})$ be a measurable space satisfying Suslin property, let $Z$ be a locally compact metrizable and separable topological space and $Y$ a separable Banach space. If $P$ from $T \times Z$ into closed and convex subsets of $Y$ is $\mathcal{M} \otimes \beta(X)$-measurable and lsc in $z$, then the map $t \rightarrow P(t)=\{\phi \in$ $C(Z, Y): \phi(z) \in P(t, z)$ for each $z \in Z\}$ is $\mathcal{M}$-measurable set-valued map from $T$ into closed and convex subsets of $C(Z, Y)$.

\section{Carathéodory convex selections}

Throughout this section $(T, \mathcal{M})$ stands for a measurable space satisfying Suslin property, $X$ denotes a locally compact Banach space (i.e. $X$ is finitedimensional by Riesz Theorem) and $Y$ is a separable Banach lattice with an order unit $e$. Since $X$ is locally compact, then there exists a sequence $\left(X_{m}\right)_{m \in \mathbb{N}}$ of relatively compact and open subsets of $X$ such that $X=\bigcup_{m=1}^{\infty} X_{m}$ and for each $m \in \mathbb{N} \bar{X}_{m} \subset X_{m+1}$.

For every $m \in N$ define the function $f_{m}: C(X, Y) \rightarrow Y$ by the formula:

$$
f_{m}(u)=\sup _{x_{1}, x_{2} \in X_{m}, \lambda \in[0,1]}\left\{u\left(\lambda x_{1}+(1-\lambda) x_{2}\right)-\left[\lambda u\left(x_{1}\right)+(1-\lambda) u\left(x_{2}\right)\right]\right\} .
$$

Lemma 3.1. For every $m \in N$ the function $f_{m}$ is continuous.

Proof. Let $\widetilde{X}_{m}=\bar{X}_{m} \times \bar{X}_{m} \times[0,1]$. For $\widetilde{x}=\left(x_{1}, x_{2}, \lambda\right) \in \widetilde{X}_{m}$ we take $\|\widetilde{x}\|=\left\|x_{1}\right\|+\left\|x_{2}\right\|+\lambda$ and define $v_{u}^{m}: \widetilde{X}_{m} \rightarrow Y$ by the formula:

$$
v_{u}^{m}(\widetilde{x})=u\left(\lambda x_{1}+(1-\lambda) x_{2}\right)-\left[\lambda u\left(x_{1}\right)+(1-\lambda) u\left(x_{2}\right)\right] .
$$

By the continuity of $u$ it is easy to verify that $v_{u}^{m} \in C\left(\widetilde{X}_{m}, Y\right)$.

We have $f_{m}(u)=\sup _{\widetilde{x} \in \widetilde{X}_{m}} v_{u}^{m}(\widetilde{x})$. Since $Y$ is a Banach lattice with an order unit, then every compact subset of $Y$ admits supremum (see e.g. [17, Proposition 2.3.10]). But the set $v_{u}^{m}\left(\widetilde{X}_{m}\right)$ is compact by the continuity of $v_{u}^{m}$ and therefore the function $f_{m}$ is well defined.

Let us take arbitrary elements $u, u_{0} \in C(X, Y)$. Define sets $A, B \in Y$ by the formulas

$$
A=\left\{v_{u}^{m}(\widetilde{x}): \widetilde{x} \in \widetilde{X}_{m}\right\} \quad \text { and } \quad B=\left\{v_{u_{0}}^{m}(\widetilde{x}): \widetilde{x} \in \widetilde{X}_{m}\right\}
$$

and assume

$$
\left\|v_{u}^{m}-v_{u_{0}}^{m}\right\|_{C\left(\widetilde{X}_{m}, Y\right)}=\sup _{\widetilde{x} \in \widetilde{X}_{m}}\left\|v_{u}^{m}(\widetilde{x})-v_{u_{0}}^{m}(\widetilde{x})\right\|<\delta .
$$


We have to show that $\|\sup A-\sup B\|<\varepsilon$. Let $\varepsilon>0$ be arbitrary taken, let $n$ be such great that $3 / n<\varepsilon$ and take $\delta<1 / n$. Let $C=A \cup B$. Since $C$ is compact in $Y$, then there exists a sequence $\left(x_{k}\right)$ in $C$ with the following property:

- for every $n \in \mathbb{N}$ there exists $N_{n}$ such that $C \subset \bigcup_{k=1}^{N_{n}} B\left(x_{k}, 1 / n\right)$, where $B\left(x_{k}, 1 / n\right)$ denotes the ball in $Y$ of a radius $1 / n$ centered at $x_{k}$.

Let $\mathcal{W}$ be a class of all finite subsets of the set $\left\{1, \ldots, N_{n}\right\}$. Choose a set $W=\left\{k_{1}, \ldots, k_{r}\right\} \in \mathcal{W}$ such that:

(i) for every $x \in C$ there exists $k_{j} \in W$ such that $x \in B\left(x_{k_{j}}, 1 / n\right)$,

(ii) for every $k_{j} \in W B\left(x_{k_{j}}, 2 / n\right) \cap A \neq \emptyset$ and $B\left(x_{k_{j}}, 2 / n\right) \cap B \neq \emptyset$.

Such $W$ exists because from the assumption it follows that for every $x \in A$ there exists $y \in B$ with $\|x-y\|<\delta<1 / n$ and for every $y \in B$ there exists $x \in A$ with $\|x-y\|<\delta<1 / n$.

From (i) we deduce that for every $x \in C$ there exists $1 \leq j \leq r$ such that $x \preceq x_{k_{j}}+1 / n \cdot e$. Therefore, every $x$ from $C$ satisfies

$$
x \preceq \sup \left\{x_{k_{j}}: 1 \leq j \leq r\right\}+1 / n \cdot e .
$$

For this reason

$$
\sup A \preceq \sup \left\{x_{k_{j}}: 1 \leq j \leq r\right\}+1 / n \cdot e
$$

and

$$
\sup B \preceq \sup \left\{x_{k_{j}}: 1 \leq j \leq r\right\}+1 / n \cdot e .
$$

On the other hand, by (ii) we conclude that that for every $1 \leq j \leq r$ there exist $x \in A$ and $y \in B$ such that

$$
x_{k_{j}} \preceq x+1 / n \cdot e \text { and } \quad x_{k_{j}} \preceq y+1 / n \cdot e \text {. }
$$

It means that

$$
x_{k_{j}} \preceq \sup A+2 / n \cdot e \quad \text { and } \quad x_{k_{j}} \preceq \sup B+2 / n \cdot e
$$

for every $1 \leq j \leq r$. Therefore,

$$
\sup _{1 \leq j \leq r} x_{k_{j}} \preceq \sup A+2 / n \cdot e \text { and } \sup _{1 \leq j \leq r} x_{k_{j}} \preceq \sup B+2 / n \cdot e .
$$

Combining above inequalities we get

$$
\sup B \preceq \sup A+3 / n \cdot e \quad \text { and } \quad \sup A \preceq \sup B+3 / n \cdot e .
$$

It means that $|\sup A-\sup B| \preceq 3 / n \cdot e$. Since $Y$ is a Banach lattice we obtain

$$
\|\sup A-\sup B\| \preceq 3 / n\|e\|<\varepsilon
$$

which means the continuity of $f_{m}$ for $m=1,2, \ldots$ 
Lemma 3.2. The set $U=\left\{u \in C(X, Y): u\left(\lambda x_{1}+(1-\lambda) x_{2}\right)-\left[\lambda u\left(x_{1}\right)+\right.\right.$ $\left.(1-\lambda) u\left(x_{2}\right)\right] \preceq 0$ for every $x_{1}, x_{2} \in X$, and each $\left.\lambda \in[0,1]\right\}$ is closed in $C(X, Y)$ and therefore, it is $\beta(C(X, Y))$-measurable.

Proof. Since $\bar{X}_{m} \subset X_{m+1}$ for each $m \in \mathbb{N}$, then

$$
\begin{aligned}
U= & \bigcap_{m=1}^{\infty}\left\{u \in C(X, Y): u\left(\lambda x_{1}+(1-\lambda) x_{2}\right)-\left[\lambda u\left(x_{1}\right)+(1-\lambda) u\left(x_{2}\right)\right] \preceq 0\right. \\
& \text { for every } \left.x_{1}, x_{2} \in X_{m} \text {, and each } \lambda \in[0,1]\right\} \\
=\bigcap_{m=1}^{\infty}\{u \in C(X, Y): & \left.\sup _{x_{1}, x_{2} \in X_{m}, \lambda \in[0,1]}\left\{u\left(\lambda x_{1}+(1-\lambda) x_{2}\right)-\left[\lambda u\left(x_{1}\right)+(1-\lambda) u\left(x_{2}\right)\right]\right\} \preceq 0\right\} .
\end{aligned}
$$

The existence of the supremum in the above formula follows from the first part of the proof of Lemma 3.1. Then

$$
U=\bigcap_{m=1}^{\infty}\left\{u \in C(X, Y): f_{m}(u) \preceq 0\right\}=\bigcap_{m=1}^{\infty} f_{m}^{-1}\left(-K_{Y}^{+}\right) .
$$

Since the positive cone in $\mathrm{Y}$ is closed ([20, Proposition 5.2]), then the set $U$ is closed in $C(X, Y)$ by Lemma 3.1. Being closed, $U$ is $\beta(C(X, Y))$-measurable.

Lemma 3.3. Let $F: T \times X \rightarrow \mathrm{Cl}$ Conv $Y$ be $\mathcal{M} \otimes \beta(X)$-measurable with orderbounded and order-convex values. Assume that $F(t, \cdot)$ is upper separated, majorized in a neighbourhood of some point $x_{1}$ and lsc for every $t \in T$. Define a set-valued function $\Phi: T \rightarrow 2^{C(X, Y)}$ by

$$
\Phi(t)=\{u \in C(X, Y): u(\cdot) \text { is an order convex selection of } F(t, \cdot)\} \text {. }
$$

Then the graph of $\Phi$ is an $\mathcal{M} \otimes \beta(C(X, Y))$ measurable set in $T \times C(X, Y)$.

Proof. The set $\Phi(t)$ is nonempty for every $t \in T$. Indeed, $F(t, \cdot)$ admits an order convex selection by Theorem 2.8 , this selection is majorized in some $U_{x_{1}}$ and therefore, it is continuous by [16, Theorem 3.1]. We have

$$
\begin{array}{r}
\Phi(t)=\{u \in C(X, Y): u(\cdot) \in F(t, \cdot)\} \cap\{u \in C(X, Y): u \text { is order convex }\} \\
=\Phi_{1}(t) \cap \Phi_{2}(t) .
\end{array}
$$

Using Lemma 2.11 we deduce that $\Phi_{1}: T \rightarrow C(X, Y)$ is $\mathcal{M}$-measurable with closed values in a Polish space $C(X, Y)$. Then the set $\operatorname{Gr}\left(\Phi_{1}\right)$ is $\mathcal{M} \otimes \beta(C(X, Y))$ measurable by [10, Theorem 3.5].

A function $u \in C(X, Y)$ is order convex if and only if $u\left(\lambda x_{1}+(1-\lambda) x_{2}\right)-$ $\left[\lambda u\left(x_{1}\right)+(1-\lambda) u\left(x_{2}\right)\right] \preceq 0$ for every $x_{1}, x_{2}$ in $X$ and $\lambda \in[0,1]$. This is equivalent to the fact that $u \in U$, where $U$ is a set defined in Lemma 3.2. Then $\Phi_{2}(t) \equiv U$ 
for every $t \in T$ and therefore, $\operatorname{Gr}\left(\Phi_{2}\right)=T \times U$ is $\mathcal{M} \otimes \beta(C(X, Y))$ measurable by Lemma 3.2. Finally, $\operatorname{Gr}(\Phi)=\operatorname{Gr}\left(\Phi_{1}\right) \cap \operatorname{Gr}\left(\Phi_{2}\right)$, which completes the proof. $\square$

TheOREM 3.4. Let $(T, \mathcal{M})$ be a measurable space satisfying Suslin property, let $X$ be a locally compact Banach space and let $Y$ be a separable Banach lattice with an order unit e. Let $F: T \times X \rightarrow \mathrm{Cl}$ Conv $Y$ be $\mathcal{M} \otimes \beta(X)$-measurable with order-convex and order-bounded values. Assume that $F(t, \cdot)$ is upper separated, majorized in a neighbourhood of some point $x_{1} \in X$ and lsc for every $t \in T$. Then there exists a selection $f$ of $F$ satisfying:

(a) $f(\cdot, x)$ is $\mathcal{M}$-measurable for all $x \in X$,

(b) $f(t, \cdot)$ is order-convex for every $t \in T$.

Moreover, the function $f(t, \cdot)$ is locally Lipschitz for every $t \in T$.

Proof. Let $\Phi$ be defined as in Lemma 3.3. By Theorem 2.9 we deduce that $\Phi$ admits a measurable selection $\phi: T \rightarrow C(X, Y)$.

Define a function $f: T \times X \rightarrow Y$ by the formula $f(t, x)=\phi(t)(x)$. We will show that $f$ is a required selection of $F$.

Given any $\bar{x} \in X$ and any open set $U \in Y$ let $\mathcal{U}=\{u \in C(X, Y): u(\bar{x}) \in U\}$. Then

$$
(f(\cdot, \bar{x}))^{-1}(U)=\{t \in T: f(t, \bar{x}) \in U\}=\{t \in T: \phi(t) \in \mathcal{U}\}=\phi^{-1}(\mathcal{U}) .
$$

Since $\mathcal{U}$ is open in $C(X, Y)$ and $\phi$ is measurable, then $\phi^{-1}(\mathcal{U}) \in \mathcal{M}$ which proves the property (a).

Moreover, since for every $t \in T \phi(t) \in \Phi(t)$, then $\phi(t)(\cdot)$ is an order-convex selection of $F(t, \cdot)$.

Now we prove the last statement of theorem. Since $f(t, x)$ is majorized in a neighbourhood $U_{x}$ of $x$ and order bounded there, then $f(t, \cdot)$ is locally orderLipschitz in $X$ by Theorem 3.2 of [16]. It means that for every $t \in T$ and $x \in X$ there exists a neighbourhood $U_{x}$ such that $\left|f\left(t, z_{1}\right)-f\left(t, z_{2}\right)\right| \preceq y\left\|z_{1}-z_{2}\right\|$ for some $y \in K^{+}$and every $z_{1}, z_{2} \in U_{x}$. Since $Y$ is a Banach lattice, we get $\left\|f\left(t, z_{1}\right)-f\left(t, z_{2}\right)\right\| \leq\|y\|\left\|z_{1}-z_{2}\right\|$ which completes the proof.

We are able to skip the conditions " $F(t, x)$ is majorized in the neighbourhood of $x_{1}$ " and " $F$ takes on order bounded values" in the case of Carathéodory setvalued functions with compact values. The following version of the Theorem 3.4 holds:

Corollary 3.5. Let $(T, \mathcal{M})$ be a measurable space satisfying Suslin property, let $X$ be a locally compact Banach space and let $Y$ be a separable Banach lattice with an order unit e. Let $F: T \times X \rightarrow \operatorname{Comp} \operatorname{Conv} Y$ be a Carathéodory setvalued function with order-convex values. Assume that $F(t, \cdot)$ is upper separated for every $t \in T$. Then there exists a selection $f$ of $F$ satisfying:

(a) $f(\cdot, x)$ is $\mathcal{M}$-measurable for all $x \in X$, 
(b) $f(t, \cdot)$ is order-convex and locally Lipschitz for every $t \in T$.

Proof. Let $\Phi$ be defined as in Lemma 3.3. Let us claim that the set $\Phi(t)$ is nonempty for every $t \in T$. Indeed, let $t \in T$ be arbitrary fixed. Every point $x \in X$ admits an open and relatively compact neigbourhood $U_{x}$ because of the local compactness of $X . F(t, \cdot)$ is continuous and compact-valued and therefore, the set $F\left(t, \bar{U}_{x}\right)$ is compact in $Y$ for every $x \in X$ (e.g. [12, Proposition 2.2.3]). Since $Y$ has an order unit, then this set admits a supremum by [17, Proposition 2.3.10]. It means that $F(t, x)$ is majorized in a neighbourhood of $x$. Then it is order-bounded there. Now, we deduce that $F(t, \cdot)$ posesses for every $t \in T$ an order-convex and continuous selection. From Theorem 2.10 it follows that $F$ is $\mathcal{M} \otimes \beta(X)$-measurable and the rest of the proof is the same as the proof of the Theorem 3.4 .

\section{REFERENCES}

[1] J.P. Aubin and A. Cellina, Differential Inclusions, Noordhoff, Leyden, 1984.

[2] S. Belov And V. Chistyakov, A selection principle for mappings of bounded variation, J. Math. Anal. Appl. 249 (2000), 351-366.

[3] C. Castaing, Sur l'existence des sections séparément mesurables et séparément continues d'une multi-application, Séminaire d'Analyse Convexe, Univ. des Sci. et Techniques du Languedoc Montpellier, 1975, Expose No. 14.

[4] A. Cellina, A selection theorem, Rend. Sem. Mat. Univ. Padova 55 (1976), 143-149.

[5] V. Chistyakov, On mappings of bounded variation, J. Dynam. Control Syst. 3 (1997), $261-289$.

[6] V. Chistyakov and A. Nowak, Regular Carathéodory-type selectors under no convexity assumptions, J. Funct. Anal. 225 (2005), 247-262.

[7] R. Fierro, C. Martinez and C. Morales, Carathéodory selections for multivalued mappins, Nonlinear Anal. 64 (2006), 1229-1235.

[8] A. Fryszkowski, Carathéodory type selectors of set-valued maps of two variables, Bull. Pol. Acad. Sci. ser. Math. 25 (1976), 41-46.

[9] H. Hermes, On continues and measurable selections and the existence of solutions of generalized differential equations, Proc. Amer. Math. Soc. 29 (1971), 535-542.

[10] C.J. Himmelberg, Measurable relations, Fund. Math. 87 (1975), 53-72.

[11] A.D. IofFe, Single valued representation of set-valued mappings II; Application to differential inclusions, SIAM J. Control Optim. 21 (1983), 641-651.

[12] M. Kisielewicz, Differential Inclusions and Optimal Control, Kluwer Acad. Publ. and Polish Sci. Publ., Warszawa-Dordrecht, 1991.

[13] A. Kucia, On the existence of Carathéodory selectors, Bull. Pol. Acad. Sci. Ser. Math. 32 (1984), 233-241.

[14] S.J. Leese, Multifunctions of Souslin type, Bull. Austral. Math. Soc. 11 (1974), 395-411.

[15] M. Michta And J. Motyl, Locally Lipschitz selections in Banach lattices, Nonlinear Anal. 71 (2009), 2335-2342.

[16] N.S. PApageorgiou, Nonsmooth analysis on partially ordered vector spaces: part 1 convex case, Pacific J. Math. 107 (1983), 403-458. 
[17] A.L. Peressini, Ordered Topological Vector Spaces, Harper and Row publishers, New York-Evanston-London, 1967.

[18] B. RICCERI, Carathéodory's selections for multifunctions with nonseparable range, Rend. Sem. Mat. Univ. Padova 67 (1982), 185-190.

[19] L. Rybiński, On Carathéodory type selections, Fund. Math. 75 (1984), 187-193.

[20] H.H. Schaefer, Banach Lattices and Positive Operators, Die Grundlehren der mathematischen Wissenschaften in Einzeldartstellungen, Band 215, Springer Verlag, BerlinHeidelberg-New York, 1974.

Manuscript received July 27, 2010

JERZY MOTYL

Faculty of Mathematics

Computer Science and Econometrics

University of Zielona Góra

Szafrana $4 \mathrm{a}$

65-516 Zielona Góra, POLAND

E-mail address: j.motyl@wmie.uz.zgora.pl 